\title{
CKI and CKII mediate the FREQUENCY-dependent phosphorylation of the WHITE COLLAR complex to close the Neurospora circadian negative feedback loop
}

\author{
Qun He, ${ }^{1,2}$ Joonseok Cha, ${ }^{1}$ Qiyang He, ${ }^{1,3}$ Heng-Chi Lee, ${ }^{1}$ Yuhong Yang, ${ }^{1,4}$ and Yi Liu ${ }^{1,5}$ \\ ${ }^{1}$ Department of Physiology, The University of Texas Southwestern Medical Center, Dallas, Texas 75390, USA; ${ }^{2}$ State Key \\ Laboratory for Agro-Biotechnology, College of Biological Sciences, China Agricultural University, Beijing 100094, China
}

The eukaryotic circadian oscillators consist of circadian negative feedback loops. In Neurospora, it was proposed that the FREQUENCY (FRQ) protein promotes the phosphorylation of the WHITE COLLAR (WC) complex, thus inhibiting its activity. The kinase(s) involved in this process is not known. In this study, we show that the disruption of the interaction between FRQ and CK-1a (a casein kinase I homolog) results in the hypophosphorylation of FRQ, WC-1, and WC-2. In the $c k-1 a^{L}$ strain, a knock-in mutant that carries a mutation equivalent to that of the Drosophila $d b t^{L}$ mutation, FRQ, WC-1, and WC-2 are hypophosphorylated. The mutant also exhibits $\sim 32 \mathrm{~h}$ circadian rhythms due to the increase of FRQ stability and the significant delay of FRQ progressive phosphorylation. In addition, the levels of WC-1 and WC-2 are low in the $c k-1 a^{L}$ strain, indicating that CK-1a is also important for the circadian positive feedback loops. In spite of its low accumulation in the $c k-1 a^{L}$ strain, the hypophosphorylated WCC efficiently binds to the C-box within the frq promoter, presumably because it cannot be inactivated through FRQ-mediated phosphorylation. Furthermore, WC-1 and WC-2 are also hypophosphorylated in the $c k a^{R I P}$ strain, which carries the disruption of the catalytic subunit of casein kinase II. In the $c k a^{R I P}$ strain, WCC binding to the C-box is constantly high and cannot be inhibited by FRQ despite high FRQ levels, resulting in high levels of frq RNA. Together, these results suggest that CKI and CKII, in addition to being the FRQ kinases, mediate the FRQ-dependent phosphorylation of WCs, which inhibit their activity and close the circadian negative feedback loop.

[Keywords: Circadian clock; casein kinase I; knock-in; ChIP; DNA binding; degradation]

Supplemental material is available at http://www.genesdev.org.

Received June 27, 2006; revised version accepted July 27, 2006.

The eukaryotic circadian clocks are composed of autoregulatory circadian negative feedback loops including both positive and negative elements (Dunlap 1999; Reppert and Weaver 2001; Young and Kay 2001; Sehgal 2004). In Neurospora, Drosophila, and mammals, the positive elements are all heterodimeric complexes, consisting of two PER-ARNT-SIM (PAS) domain-containing transcriptional factors that bind to the cis-elements in the promoter of the negative elements to activate their

Present addresses: ${ }^{3}$ Institute of Medicinal Biotechnology, Chinese Academy of Medical Sciences and Peking Union Medical College, Beijing China 100050; ${ }^{4}$ Division of Nephrology, The University of Texas Southwestern Medical Center, Dallas, Texas 75390

${ }^{5}$ Corresponding author.

E-MAIL Yi.Liu@UTsouthwestern.edu; FAX (214) 645-6049.

Article is online at http://www.genesdev.org/cgi/doi/10.1101/gad.1463506. transcription. On the other hand, the negative elements repress their own transcription by inhibiting the activity of the positive elements through their physical interactions. It is unclear how negative elements inhibit the activity of positive elements to close the circadian negative feedback loops. Since the identification of the Drosophila doubletime ( $\mathrm{dbt}$ ) gene, which encodes for a casein kinase I (CKI) homolog, it has become clear that post-translational protein phosphorylation is essential for the function of circadian clocks (Kloss et al. 1998; Price et al. 1998). Despite the evolutionary distance, remarkable conservation of post-translational regulation exists among different eukaryotic systems from fungi to human (see Discussion; Liu 2005; Heintzen and Liu 2006).

In the filamentous fungus Neurospora crassa, the core circadian negative feedback loop consists of four essen- 
tial components: WHITE COLLAR-1 (WC-1), WC-2, FREQUENCY (FRQ), and a FRQ-interacting RNA hecliase FRH. WC-1 and WC-2, two PAS domain-containing transcription factors, form a heterodimeric WC complex (D-WCC) in the dark, which binds to the Clock (C)-box in the frq promoter to activate $f_{r q}$ transcription (Crosthwaite et al. 1997; Cheng et al. 2001b, 2002; Loros and Dunlap 2001; Froehlich et al. 2003; He and Liu 2005). Thus, WCC is the positive element in the Neurospora circadian negative feedback loop. On the other hand, FFC, the complex formed by FRQ and FRH functions as the negative element (Cheng et al. 2005). To repress the transcription of $f r q$, FFC possibly mediates the inhibition of the WCC activity through their physical interaction (Aronson et al. 1994; Merrow et al. 1997, 2001; Cheng et al. 2001a, 2003; Denault et al. 2001; Froehlich et al. 2003). In a wild-type strain, this circadian negative feedback loop generates robust daily rhythms of frq RNA and FRQ protein (Garceau et al. 1997). When the circadian negative feedback loop is disrupted by mutation of $f r q$ or down-regulation of frh, frq RNA levels stay at constant higher levels, resulting in arrhythmicities (Aronson et al. 1994; Cheng et al. 2005). In addition to their role in the circadian negative feedback loop in the dark, WC-1and WC-2 are also essential components for the light responses and light resetting of the clock, with WC-1 being the blue-light photoreceptor (Crosthwaite et al. 1997; Froehlich et al. 2002; He et al. 2002; He and Liu 2005).

In addition to the repression of D-WCC activity in the dark, FRQ promotes the accumulation of WC-1 and WC-2, forming positive feedback loops that are interlocked with the negative loop (Lee et al. 2000; Cheng et al. 2001b, 2003; Merrow et al. 2001), a feature that is shared by animal circadian systems (Glossop et al. 1999; Shearman et al. 2000). In Neurospora, these positive feedback loops have been shown to be important for the robustness and function of the clock (Cheng et al. 2001b; Schafmeier et al. 2006). It was recently shown that the phosphorylation of the PEST-2 region of cytoplasmic FRQ is important for the accumulation of WC-1 but not WC-2 (Schafmeier et al. 2006).

FRQ, WC-1, and WC-2 are regulated by phosphorylation events. After its synthesis, FRQ is immediately phosphorylated and becomes progressively more phosphorylated over time before its degradation through the ubiquitin-proteasome pathway mediated by FWD-1 (Garceau et al. 1997; He et al. 2003, 2005a). Thus, in the dark, FRQ is not only robustly rhythmic in quantity, but also in its phosphorylation states. CK-1a (casein kinase 1a), CKII (casein kinase II), and CAMK-1 are the three identified FRQ kinases (Gorl et al. 2001; Yang et al. 2001, 2002, 2003). However, only CKII's role in mediating FRQ phosphorylation is firmly established in vivo (Yang et al. 2002, 2003). In vitro, CKII is one of the main kinases that phosphorylate FRQ. In strains in which either the CKII catalytic subunit $(c k a)$ or one of its regulatory subunits (ckb1) is disrupted, FRQ is both hypophosphorylated and more stable, and the clock function is either completely abolished (cka mutant) or oscillates with a severely damped amplitude (ckb1 mutant). Furthermore, in the cka mutant strain that has no CKII activity, frq mRNA levels are constantly high, which is reminiscent of the frq RNA levels in strains with a disrupted circadian negative feedback loop (Yang et al. 2002). These data suggest that CKII not only promotes FRQ degradation, but it is also required for the repressor function of FRQ. The mechanism by which CKII carries this latter function is not known.

CK-1a is one of the two Neurospora CKI homologs and it can phosphorylate the PEST-1 and PEST-2 domains of FRQ in vitro (Gorl et al. 2001). The deletion of the PEST-1 domain resulted in the increased stability of FRQ and a long period rhythm. More importantly, CK-1a was found to associate with FRQ, suggesting that it may phosphorylate FRQ in vivo (Gorl et al. 2001; Cheng et al. 2005). However, in vivo evidence for the involvement of CK-1a in the clock was not available because CK-1a is essential for cell survival in Neurospora.

Similar to FRQ, both WC-1 and WC-2 are phosphorylated both in the dark and in a light-dependent manner. Their phosphorylation plays important roles in regulating WCC activity (He and Liu 2005; He et al. 2005b; Schafmeier et al. 2005, 2006). We previously identified five major in vivo WC-1 phosphorylation sites, located immediately downstream from its DNA-binding domain (He et al. 2005b). Mutation of these light-independent sites suggested that they are critical for circadian clock function and they negatively regulate the D-WCC activity. The importance of WC phosphorylation in the circadian clock was later confirmed by the surprising observation by Schafmeier et al. (2005) that the WC phosphorylation is FRQ dependent. In the frq-null strain, both WC-1 and WC-2 are hypophosphorylated. In a wildtype strain, WC-2 exhibits a robust circadian rhythm of its phosphorylation profile when analyzed on two-dimensional electrophoresis. Importantly, the activation of $f_{r} q$ transcription correlates with the hypophosphorylation of the WCs. Consistent with these data, we showed that dephosphorylation of the Neurospora WCC significantly promotes its binding to the C-box (He and Liu 2005). Together, these results suggest a model in which FFC inhibits the WCC activity by promoting the phosphorylation of WC proteins. Interestingly, PER-dependent phosphorylation of CLK was recently also observed in Drosophila (Kim and Edery 2006; Yu et al. 2006), suggesting a common mechanism that closes the circadian negative feedback loops.

The kinase(s) recruited by FRQ to phosphorylate the WC proteins has not been identified. How WCC activity is affected by the phosphorylation mediated by this kinase(s) is also not known. In this study, we show that like CKII, CK-1a phosphorylates FRQ in vivo to promote its degradation. More importantly, both kinases mediate the FRQ-dependent phosphorylation of WCC, which inhibits its activity to close the circadian negative feedback loop. In addition to CK-1a's role in the negative feedback loop, it is also required for the function of the circadian positive feedback loops by increasing WC levels. 
He et al.

\section{Results}

\section{Disruption of the FRQ/CK-1a interaction results in} hypophosphorylation of FRQ

To understand the in vivo function of CK-1a in the Neurospora clock, we investigated the functional importance of the FRQ/CK-1a interaction. We first mapped the domain on FRQ that is required for the FRQ/CK-1a interaction. Figure 1A shows the domain structure of FRQ. The coiled-coil (CC) and FFD domains were previously shown to mediate the FRQ-FRQ or FRQ-FRH interaction, respectively (Cheng et al. 2001a, 2005). S513 is one of the previously identified FRQ phosphorylation sites that mediates the degradation of FRQ (Liu et al. 2000). PEST-1 and PEST-2 are two PEST domains that can be phosphorylated by CK-1a in vitro /Gorl et al. 2001; Schafmeier et al. 2006). To study FRQ/CK-1a interaction, we introduced a construct (pMyc-CK-1a) expressing Myc-tagged CK-1a (under the quinic acid inducible promoter) into a wild-type strain and a series of small FRQ (sFRQ) mutant strains (in $f r q^{10}$ [frq-null] background), which express various internal in-frame deletions of the sFRQ form covering the entire sFRQ ORF (Fig. 1A; Liu et al. 2000; Cheng et al. 2005). Western blot analysis showed the expression of Myc-CK-1a as triplet bands (Fig. 1B), possibly either due to alternative protein initiation or post-translational modification of CK-1a. Immunoprecipitation using c-Myc monoclonal antibody showed that Myc-CK-1a specifically interacts with the wild-type FRQ and all sFRQ forms except for sFRQ4 (Fig. 1A,B; Supplementary Fig. 1). These data indicate that the protein region deleted in sFRQ4, but not other parts of FRQ, is required for FRQ/CK-1a interaction.

Interestingly, FRQ in sFRQ4 was previously shown to be hypophosphorylated and more stable (Liu et al. 2000; Gorl et al. 2001). The FRQ region deleted in sFRQ4 contains the three identified FRQ phosphorylation sites (T501, S513, and S519) and the PEST-1 domain (Fig. 1C). Further deletion analyses revealed that an $\sim 63$-aminoacid (amino acids 470-532) region upstream of PEST-1 is required for the FRQ/CK-1a interaction (Fig. 1C; Supplementary Fig. 1). The amino acid sequence alignment of this region, named FCD (for FRQ/CK-1a interaction domain), among various fungal FRQ homologs, shows that this is one of the most conserved regions in the FRQ ORF, suggesting its importance in FRQ function.

FCD contains no F-X-X-X-F motif, which was previously identified as a CK1 docking site in mammalian mPER and NFAT proteins (Okamura et al. 2004). This suggests that distinct docking sites exist in mediating CKI's interaction with FRQ and mPERs. The three known FRQ phosphorylation sites reside in the C-terminal part of FCD, which is immediately upstream of the PEST-1 domain. This suggests that CK-1a may bind to the N-terminal part of this domain and phosphorylate FRQ at these sites. To test this hypothesis, we mutated
Figure 1. Disruption of the FRQ/CK-1a results in the hypophosphorylation of FRQ. (A) Graphic diagrams showing the domain structure of FRQ and different sFRQ internal deletion mutants. (CC) Coiled-coil domain; (FFD) FRQ-FRH interaction domain; (S513) a previously identified FRQ phosphorylation site. FRQ amino acids deleted in each mutant are indicated. $(B)$ Immunoprecipitation assay showing that sFRQ4 failed to interact with Myc-CK-1a. The asterisk indicates the IgG signal. $(C)$ Graphic diagrams showing FRQ/CK-1a interaction domain (FCD) and the internal deletions of FRQ in various mutants. The black bars indicate the regions deleted in the different mutants. FRQ amino acids deleted in each mutant are indicated. Except for FRQ4A1, which expresses full-length FRQ protein with the indicated deletion, other mutants only express sFRQ. The amino acid alignment of the FCD domains of different FRQ homologs is shown at bottom and the identical residues among different homologs are boxed. (Neu) Neurospora crassra; (Mag) Magnaporthe grisea; (Sor) Sordaria fimicola; (Lep) Leptosphaeria australiensis; (Gib) Gibberella zeae; (Hyp) Hypocrea spinulosa. In the 4A11 and 4A12 mutants (express full-length FRQ), the amino acids indicated by the asterisks were mutated to alanines. (p) The three previously identified phosphorylation sites. $(D)$ Immunoprecipitation assay using c-Myc antibody showing that FRQ failed to interact with MycCK-1a in the FRQ4A11 and FRQ4A12 mutants. $(E)$ Western blot analysis showing that FRQ is hypophosphorylated in the FRQ4A11 and FRQ4A12 mutants. The asterisk indicates a nonspecific band recognized by our FRQ antibody. All cultures were grown in LL.
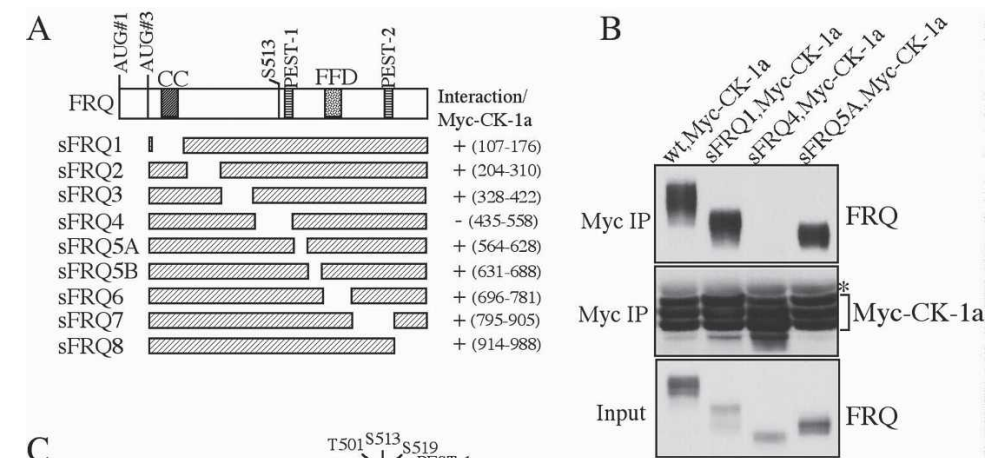

C
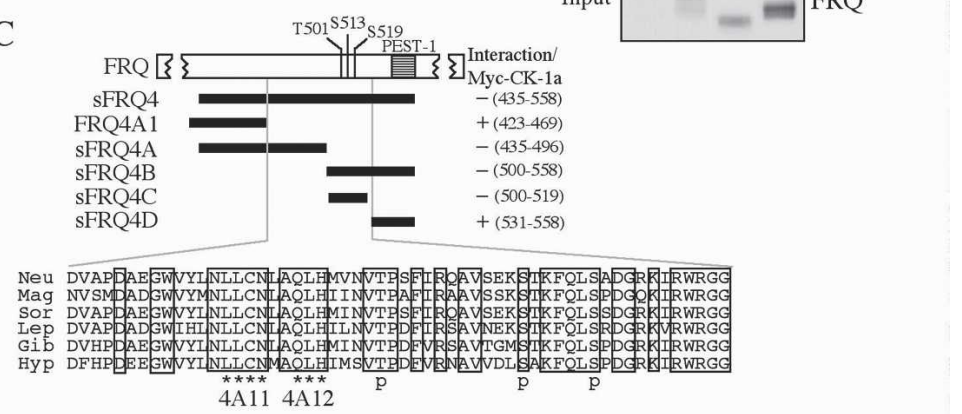

$\mathrm{D}$
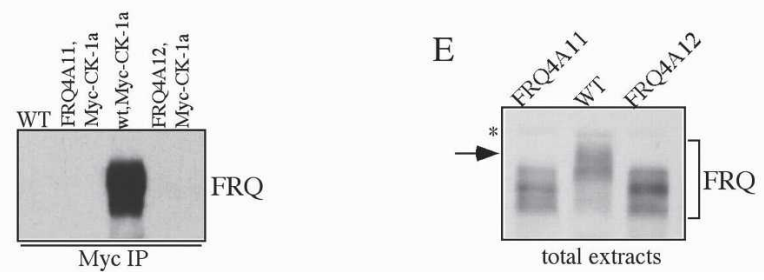
two stretches of highly conserved sequences immediately upstream of T501, amino acids 488-491 (LLCN) and amino acids 493-496 (QLH), into alanines in the full-length $f r q$ construct, respectively (Fig. 1C). Immunoprecipitation assay showed that these point mutations completely abolished the FRQ/CK-1a interaction (Fig. 1D), indicating that they are critical residues in mediating the interaction between FRQ and CK-1a.

To investigate the importance of FRQ/CK-1a interaction in FRQ phosphorylation, FRQ phosphorylation patterns in the FRQ4A11 and FRQ4A12 strains were compared with those of the wild-type strain. Cultures were grown in constant light (LL), a condition that results in the even distribution of various FRQ phosphorylated forms (Garceau et al. 1997; Liu et al. 2000). As shown in Figure 1E, FRQ in these two mutant strains was hypophosphorylated and lacked the extensively phosphorylated FRQ forms present in the wild-type strain. These data, together with previous results, suggest that CK-1a binds and phosphorylates FRQ in vivo. Several FRQ phosphorylated forms could still be observed in the mutant strains, suggesting that they can be phosphorylated by other kinases, including CKII (Yang et al. 2001, 2002, 2003).

FRQ/CK-1a interaction is required for the FRQ-dependent WC phosphorylation and important for circadian clock function

The interactions of FRQ with WCC and CK-1a suggest that WCC may associate with CK-1a through FRQ and that CK-1a may mediate the FRQ-dependent WC phosphorylation. To test this hypothesis, we examined whether WC proteins and CK-1a can form a complex in a FRQ-dependent manner. As shown in Figure 2A, immunoprecipitation of Myc-CK-1a pulled down WC-1 in a wild-type strain but not in a $f r q^{10}$ strain, indicating that WC-1 and CK-1a can complex with each other and that it is mediated by FRQ.

If CK-1a phosphorylates WC proteins through its interaction with FRQ, we expected that the disruption of FRQ/CK-1a interaction should result in the loss of FRQdependent WC phosphorylation. We found that using SDS-PAGE gels containing a ratio of 149:1 acrylamide/ bisacrylamide resulted in significantly better separation of phosphorylated WC species than the regularly used 37.5:1 SDS-PAGE gels. This is especially true for the smaller WC-2 protein (Fig. 3C). Previous studies by us and other groups have shown that various forms of WC-1 and WC-2 with different mobility shift are all due to phosphorylation events (Talora et al. 1999; He et al. 2005b; Schafmeier et al. 2005). As predicted, similar to the $f r q^{10}$ strain, both WC-1 and WC-2 are hypophosphorylated in the sFRQ4, FRQ4A11, and FRQ4A12 mutants (Fig. 2B,C), in which FCD is either deleted or mutated by point mutations. In addition, the levels of WC-1 and WC-2 in these mutants are comparable to those in the $\mathrm{rr}^{10}$ strain but are significantly lower than the wildtype strain, indicating that the positive feedback loops
A

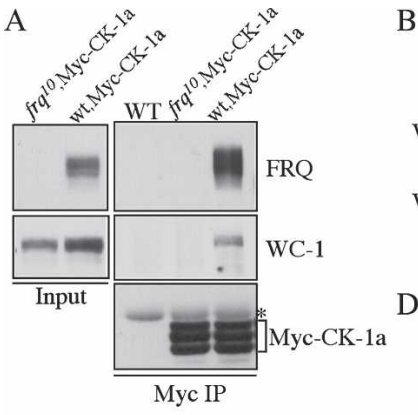

C

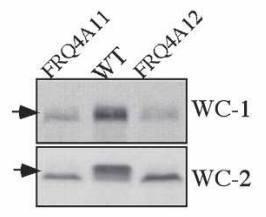

B

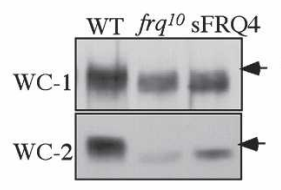

D

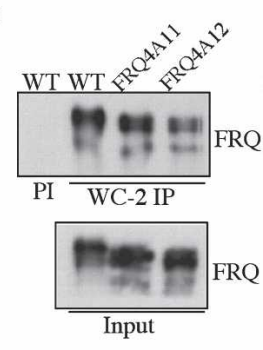

$\mathrm{E}$

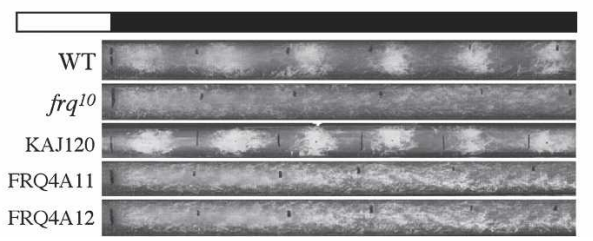

Figure 2. Disruption of the FRQ/CK-1a results in the hypophosphorylation of FRQ and arrhythmic conidiation rhythms. (A) Immunoprecipitation assay using Myc antibody showing that WC-1 forms a complex with Myc-CK-1a in the wild-type strain, but not in the fr $q^{10}$ strain. $(B, C)$ Western blot analyses showing that WC-1 and WC-2 are hypophosphorylated and at low levels in the $f r q^{10}$, sFRQ4, FRQ4A11, and FRQ4A12 strains. The arrows indicate the extensively phosphorylated WC species absent in the mutants. (D) Immunoprecipitation assay using WC-2 antibody showing that the mutant FRQ in the FRQ4A11 and FRQ4A12 strains can still interact with the WC complex. All cultures were grown in LL. (PI) Immunoprecipitation using preimmune serum. $(E)$ Race tube assays showing the arrhythmic conidiation in the FRQ4A11 and FRQ4A12 strains. KAJ120 is a $f r q^{10}$ strain containing a wild-type $f r q$ construct. The black lines indicate the daily growth fronts of the cultures.

are also impaired by the disruption of the FRQ/CK-1a interaction.

To rule out the possibility that the impairment of WC phosphorylation and low WC levels in these mutants is due to the abolition of FRQ-WCC interaction, we examined FRQ-WCC interaction in the FRQ4A11 and FRQ4A12 mutants. As shown in Figure 2D, immunoprecipitation of WC-2 pulled down FRQ in the wild-type as well as two mutant strains, indicating that FRQ-WCC interaction is independent of the FRQ/CK-1a interaction. Therefore, these results suggest that CK-1a phosphorylates WC proteins in a FRQ-dependent manner through its tight association with FRQ. In addition, the FRQ/CK-1a interaction or the WC phosphorylation mediated by such an interaction is important for FRQ's function in promoting the accumulation of $\mathrm{WC}-1$ and WC-2 proteins.

To further confirm the importance of the FRQ/CK-1a interaction, circadian conidiation rhythms of the 
He et al.

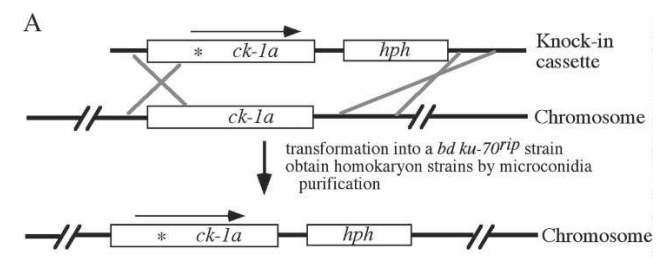

B

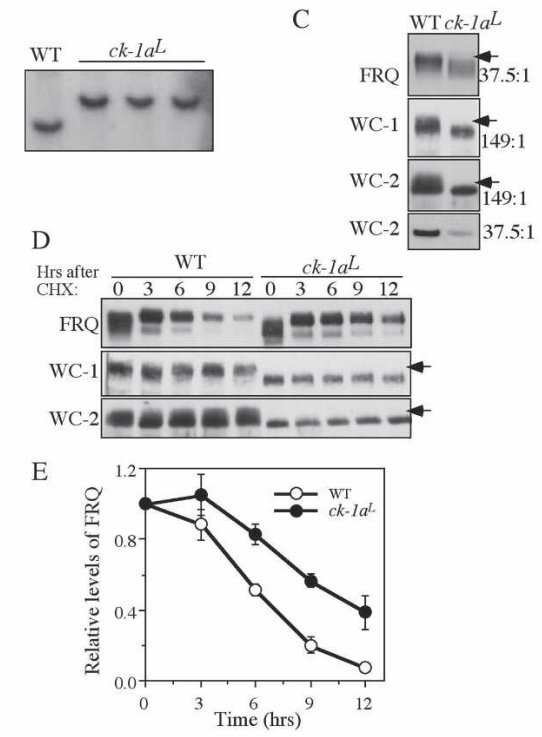

Figure 3. FRQ, WC-1, and WC-2 are hypophosphorylated in the $c k-1 a^{L}$ strain, a knock-in mutant carrying the $d b t^{L}$ mutation. (A) Graphic diagram depicting the procedures to create the homokaryotic $c k-1 a^{L}$ strain by homologous recombination (see Materials and Methods for details). The asterisk in the $c k-1 a$ ORF indicates the location of the $d b t^{L}$ mutation. (B) Southern blot analysis showing the insertion of $h p h$ in the endogenous $c k-1 a$ locus. The genomic DNA was digested by SphI and the membrane was probed with a $c k-1 a$-specific probe. $(C)$ Western blot analyses showing that hypophosphorylation of FRQ, WC-1, and WC-2 in the $c k-1 a^{L}$ strain. Cultures were grown in LL. The arrows indicate the extensively phosphorylated FRQ or WC species absent in the mutants. The numbers on the right indicate the ratio of acrylamide/bisacrylamide used in the SDS-PAGE. Note that for WC-2, the regular SDS-PAGE (37.5:1) failed to resolve different WC-2-phosphorylated forms. $(D)$ Western blot analyses showing the stability of FRQ, WC-1, and WC-2 after the addition of CHX $(10 \mu \mathrm{g} / \mathrm{mL})$ in the wild-type and $c k-1 a^{L}$ strains. Cultures were grown in LL. (E) Densitometric analysis from four independent experiments showing the degradation of FRQ in the wild-type and $c k-1 a^{L}$ strains after the addition of CHX.

FRQ4A11 and FRQ4A12 strains were examined by race tube assays. As shown in Figure 2E, similar to the $f r q^{10}$ strain, these mutants exhibited constant conidiation in constant darkness without apparent rhythmicity. Therefore, we conclude that the FRQ/CK-1a interaction is essential for the normal circadian clock function.

The creation of a Neurospora ck-1a knock-in mutant carrying the $\mathrm{dbt}^{\mathrm{L}}$ mutation

Although the results presented above suggest important roles of CK-1a in FRQ and WC phosphorylation, further confirmation of this conclusion was hindered by the lack of genetic evidence. ck-1a is an essential gene in Neurospora (Gorl et al. 2001) and we failed to obtain a homokaryotic Neurospora ck-1a knockout strain (Q. He and Y. Liu, unpubl.). The Drosophila $d b t^{L}$ mutant has a single Met to Ile mutation at a conserved Met residue in the DBT kinase domain (Kloss et al. 1998; Price et al. 1998). Such a mutation leads to reduced DBT activity and long period length of the clock (Preuss et al. 2004). Because fruit flies harboring a $d b t^{L}$ mutation were viable but displayed a strong circadian phenotype, we hoped that an equivalent mutation in Neurospora ck-1a would also be compatible with cell survival and, perhaps, affect the circadian clock. To obtain such a mutant, a knock-in cassette was made from genomic DNA that contains the mutated ck-1a gene (the conserved Met83 was changed to Ile) and a hygromycin resistance gene $(h p h)$ inserted downstream from ck-1a $3^{\prime}$ untranslated region (UTR) (Fig. 3A). This cassette was then transformed into a $b d$ ku- $70^{\text {rip }}$ strain, which is defective in nonhomologous recombination (Ninomiya et al. 2004), and hph-resistant transformants were selected. Sequencing of the endogenous $c k-1 a$ gene of the transformants was used to identify heterokaryon strains in which the mutated $c k-1 a$ cassette was integrated by homologous recombination. The homokaryotic strain with the $c k-1 a d b t^{L}$ mutation, designated as $c k-1 a^{L}$ (in bd ku-70 rip background), was then obtained by microconidia purification.

Southern blot analysis confirmed the integration of the knock-in cassette at the endogenous $c k-1 a$ locus and the homokaryotic nature of the $c k-1 a^{L}$ strains (Fig. 3B). DNA sequencing of the endogenous $c k-1 a$ gene further confirmed the mutated $c k-1 a$ in these strains (data not shown). The $c k-1 a^{L}$ strains exhibits slower growth and produces less aerial hyphae and conidia than the wildtype strain. The bd ku-70 rip strain exhibits normal growth, development, and circadian rhythms like a wildtype $b d$ strain, indicating that the ku- $70^{\text {rip }}$ mutation does not contribute to these phenotypes in the $c k-1 a^{L}$ strains (data not shown). Since hph is integrated downstream from the ck-1a 3'UTR, a region with no predicted genes, the growth and developmental phenotypes of the $c k-1 a^{L}$ strains suggest that they are due to partial function of CK-1a caused by the $d b t^{L}$ mutation.

To our knowledge, this is the first knock-in strain with a point mutation created in Neurospora. The approach we described here will be very useful in studying the functions of essential genes in this organism.

Hypophosphorylation of FRQ, WC-1, and WC-2, and reduction of FRQ degradation rate in the ck-1 $a^{L}$ strain

We then compared the phosphorylation profiles of FRQ, WC-1, and WC-2 in the wild-type and $c k-1 a^{L}$ strains for cultures grown in LL. As shown in Figure 3C, all three proteins are hypophosphorylated in the $c k-1 a^{L}$ strain. These results provide strong in vivo evidence suggesting that CK-1a phosphorylates FRQ, WC-1, and WC-2 in vivo. In addition, both the levels of WC-1 and WC-2 in 
the mutant were lower than the wild-type strain, indicating the impairment of the positive feedback loops.

Mutation of S513 in the FCD and the deletion of PEST-1 domain lead to increased FRQ stability (Liu et al. 2000; Gorl et al. 2001), suggesting that CK-1a phosphorylation of FRQ regulates its stability. To test this hypothesis, we compared the stability of FRQ in the wild-type and $c k-1 a^{L}$ strains after the addition of the protein synthesis inhibitor cycloheximide (CHX). As shown in Figure 3, D and E, FRQ degradation rate was slower in the $c k-1 a^{L}$ strain, indicating that CK-1a phosphorylation of FRQ promotes its degradation. In contrast, there is no significant difference in the degradation rates of WC-1 and WC-2 between the wild-type and $c k-1 a^{L}$ strains, suggesting that the low levels of WC-1 and WC-2 in the $c k-1 a^{L}$ strain is not simply due to changes in their stability.

Long period circadian rhythms and hypophosphorylated WC proteins in DD in the ck-1 $1 a^{L}$ strain

The slow degradation rate of FRQ in the $c k-1 a^{L}$ strain suggests that it should exhibit a long period circadian phenotype. As expected, on race tubes in constant darkness (DD), despite less conidiation than the wild-type strain, the $c k-1 a^{L}$ strain exhibits a low-amplitude circadian conidiation rhythm with a period of $\sim 32 \mathrm{~h}$ at room temperature for $\sim 6-7 \mathrm{~d}$, a period length that is $\sim 10 \mathrm{~h}$ longer than a wild-type strain (Fig. 4A). In addition, the phase of the first conidiation band after the light/dark (LD) transition was dramatically delayed in the $c k-1 a^{L}$ strain ( $19 \mathrm{~h}$ after the LD transition) compared with the wild-type strain $(\sim 10 \mathrm{~h}$ after). Under LD 12/12 cycles, despite its long period in $\mathrm{DD}$, the conidiation rhythm of the $c k-1 a^{L}$ strain could be entrained to $24 \mathrm{~h}$, but their peaks of the conidiation bands (about ZT16) were delayed for $\sim 5 \mathrm{~h}$ compared with the wild-type strain (about ZT11) (Fig. 4B).

We then examined the oscillation of FRQ in DD. In the wild-type strain, similar to previously shown, FRQ exhibited robust $\sim 22$-h rhythms both in its amount and phosphorylation profiles (Fig. 5A). In the $c k-1 a^{L}$ strain, rhythms of FRQ amounts and FRQ phosphorylation profiles were also visible but with a dramatically longer period than the wild-type. The long period FRQ oscillation in the mutant is indicated by the oscillations of FRQ amounts and FRQ phosphorylation profiles. The hypophosphorylated FRQ (the products of newly synthesized FRQ) appeared at DD16 and DD48 in the mutant, consistent with its 32 -h conidiation rhythm. In addition, the overall FRQ levels in DD in the $c k-1 a^{L}$ strain are comparable or slightly higher than those in the wild-type strain.

After the initial degradation of FRQ following the LD transition, there was a small $(<4 \mathrm{~h})$ delay in both the start of a new cycle of FRQ synthesis (indicated by hypophosphorylated FRQ in both strains at DD16) and in the peak of FRQ amounts in the mutant compared with the wildtype strain, suggesting that the transcriptional activation

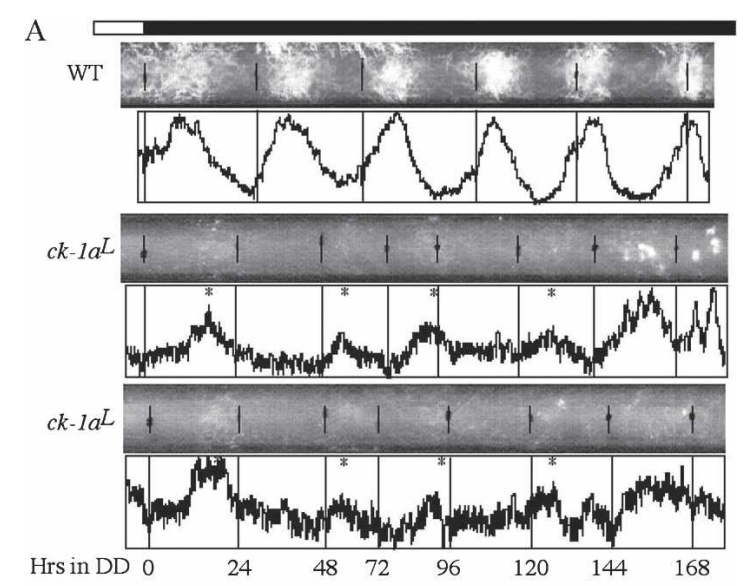

B

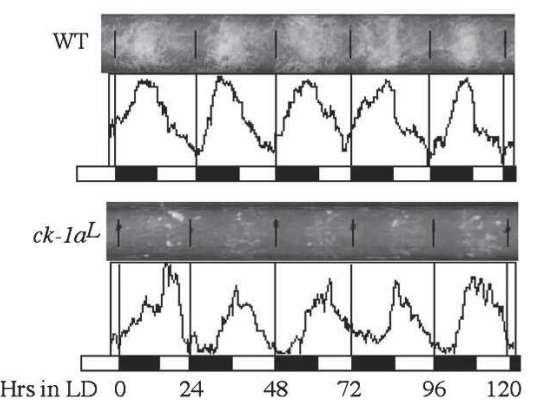

Figure 4. Race tube assays showing that the $c k-1 a^{L}$ strains exhibit a long period conidiation rhythm with a delayed phase. (A) Race tube results showing the conidiation rhythms of the wild-type and two independent $c k-1 a^{L}$ strains in DD. Densitometric results reflecting the density of conidiation are shown below the race tubes. The asterisks mark the peak of conidia bands in the $c k-1 a^{L}$ strains. (B) Race tube results showing the conidiation rhythms of the wild-type and $c k-1 a^{L}$ strains in LD (12/12) cycles.

of $f r q$ in a new cycle is not significantly delayed in the mutant. In contrast, the most significant delay of FRQ oscillation in the mutant appears to be the process of FRQ progressive phosphorylation and degradation. In the wild-type strain, FRQ with its intermediate phosphorylated forms peaked at DD20 and became hyperphosphorylated and degraded from DD24-DD32 in a process that takes $12 \mathrm{~h}$. In the $c k-1 a^{L}$ strain, however, such a process took $\sim 20 \mathrm{~h}$; the hypophosphorylated and intermediately phosphorylated FRQ at DD20 did not become hyperphosphorylated until DD40. This data suggests that the impaired CK-1a activity dramatically slows down the process of FRQ progressive phosphorylation in the $c k-1 a^{L}$ strain.

To compare the phosphorylation profiles of WC-1 and WC-2 in the wild-type and $c k-1 a^{L}$ strains in DD, we performed side-by-side comparisons for cultures harvested at different time points in DD (Fig. 5B). In addition to the phosphorylation in the dark, the phosphorylation of WC proteins can be further promoted by light exposure (Talora et al. 1999; Schwerdtfeger and Linden 2000; He and Liu 2005). Thus, WCs become hyperphosphorylated after a light pulse or in LL. For the wild-type 
He et al.

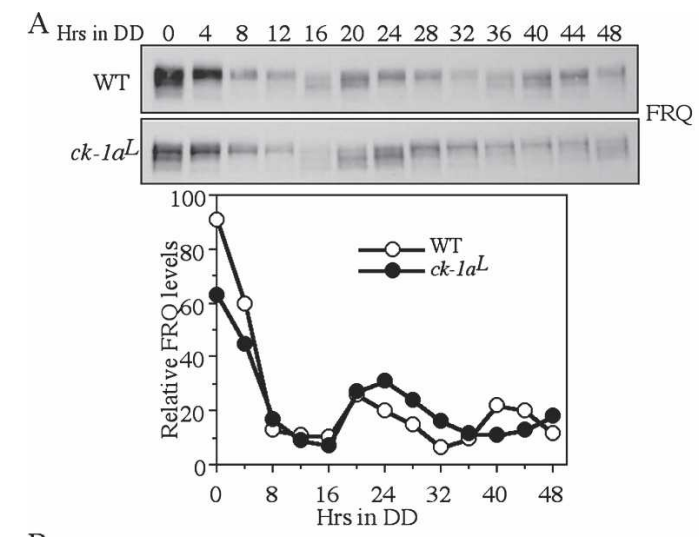

B
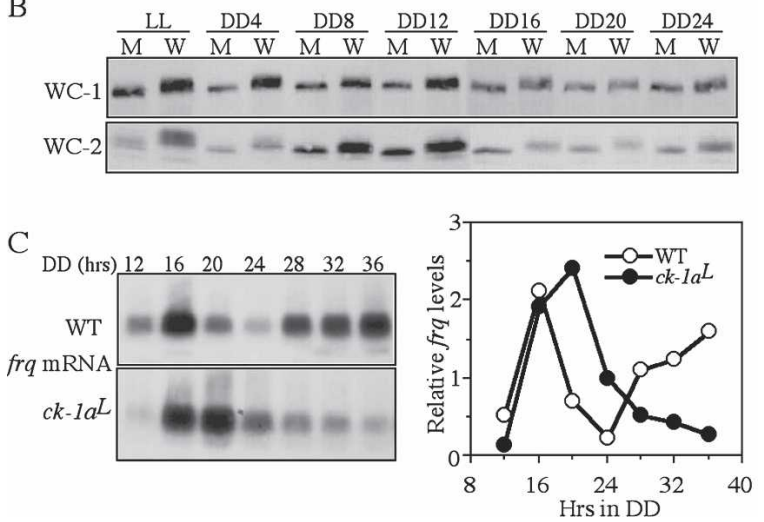

Figure 5. Long-period FRQ rhythm and hypophosphorylation of WC-1 and WC-2 in the $c k-1 a^{L}$ strain. $(A)$ Western blot analysis showing the oscillation of FRQ in the wild-type and $c k-1 a^{L}$ strains in DD. The densitometric analysis of the Western blot results is shown below. $(B)$ Western blot analysis showing sideby-side comparison of WC- 1 and WC- 2 phosphorylation profiles in the wild-type and $c k-1 a^{L}$ strains at different time points in DD. (W) The wild-type strain; (M) the $c k-1 a^{L}$ strain. (C) Northern blot analysis showing the oscillation of $f r q$ RNA in the wild-type and $c k-1 a^{L}$ strains in DD. The densitometric analysis of the Northern blot results is shown on the right.

strain, both WC-1 and WC-2 became dephosphorylated after the LD transition, probably due to the action of protein phosphatase(s) such as PP2A (Schafmeier et al. 2005) and stayed at the intermediate phosphorylationed states in DD (Fig. 5B). Rhythms of WC phosphorylation in DD were not obvious from their mobility shifts in the one-dimentional SDS-PAGE analyses due to the sensitivity limits of the method (Schafmeier et al. 2005). In the $c k-1 a^{L}$ strains, WC-1 and WC-2 were hypophosphorylated at all time points, indicating that CK-1a is a major kinase phosphorylating WC proteins in DD and LL. For the LL sample, although it is hypophosphorylated compared with the wild-type strain, some WC-2phosphorylated forms can be seen in the mutant, indicating that an additional kinase(s) is also responsible for the light-dependent WC phosphorylation. Furthermore, the levels of WC-1 and WC-2 in the mutant were lower than those in the wild-type strain in $\mathrm{DD}$, indicating the impairment of the circadian positive feedback loops when the function of CK-1a is impaired.
Despite the low levels of WC proteins in the $c k-1 a^{L}$ strain, its frq RNA levels in DD were comparable to those of the wild-type strain and exhibited a long period rhythm (Fig. 5C). Similar to the FRQ protein rhythms, there was only a small delay in the peak of $f_{r} q$ mRNA in the $c k-1 a^{L}$ strain, whereas there is a long delay in the decreasing phase of frq RNA. These results suggest that the hypophosphorylated WC proteins in the mutant may be more active in activating frq transcription than the wild-type strain, which is consistent with previous results (He and Liu 2005; He et al. 2005b; Schafmeier et al. 2005). Furthermore, the long delay in the decreasing phase of frq RNA in the $c k-1 a^{L}$ strain suggests that the circadian negative feedback loop is severely impaired despite the presence of normal or slightly higher amounts of FRQ.

\section{WCC binds to the frq C-box rhythmically in vivo}

In a wild-type Neurospora strain, the phosphorylation profiles of WC- 2 show a circadian rhythm, with the timing of WC-2 hypophosphorylation correlating with the timing of frq activation (Schafmeier et al. 2005). In vitro DNA-binding analysis previously showed that WCC's ability to bind to the frq C-box oscillates daily and the dephosphorylation of WCC increased its DNA binding (Froehlich et al. 2003; He and Liu 2005). To understand how WCC phosphorylation regulates its binding to the fr $q$ promoter in a circadian cycle, we examined the in vivo WCC binding to frq C-box in DD by semiquantitative chromatin immunoprecipitation assay (ChIP). Figure 6A shows the FRQ oscillation for samples used in the ChIP assay. As shown in Figure 6B, WCC bound to the frq C-box rhythmically, peaking at DD14 and DD36, corresponding to the time of frq mRNA peaks (Fig. 5C; Aronson et al. 1994; Garceau et al. 1997). These time points also correlate with the time when WC-2 is least phosphorylated (Schafmeier et al. 2005), suggesting that the hypophosphorylated species of WCC is its most active form. Thus, similar to the situation demonstrated in Drosophila and mouse, the positive limbs of the clock bind to the promoter of the negative elements rhythmically (Ripperger and Schibler 2006; Yu et al. 2006).

The comparison of the rhythms of FRQ expression and the WCC C-box binding showed that WCC's C-box binding peaks when FRQ protein was at its trough, suggesting that the inhibition of WCC by FRQ is released when FRQ levels are low. Furthermore, after the newly synthesized FRQ was made, WCC's binding to the frq promoter was quickly reduced (note the significant drop of binding at DD18) and stayed at a low level until the reactivation of WCC. These results indicate that the inhibition of WCC activity by FRQ is rapid in a wild-type strain, consistent with previous results (Merrow et al. 1997; Schafmeier et al. 2006).

WCC binding to the C-box cannot be efficiently inhibited by FRQ in the ck-1 $a^{L}$ strain

The hypophosphorylated WC proteins in the $c k-1 a^{L}$ strain and its long delay in the decreasing phase of $f r q$ 


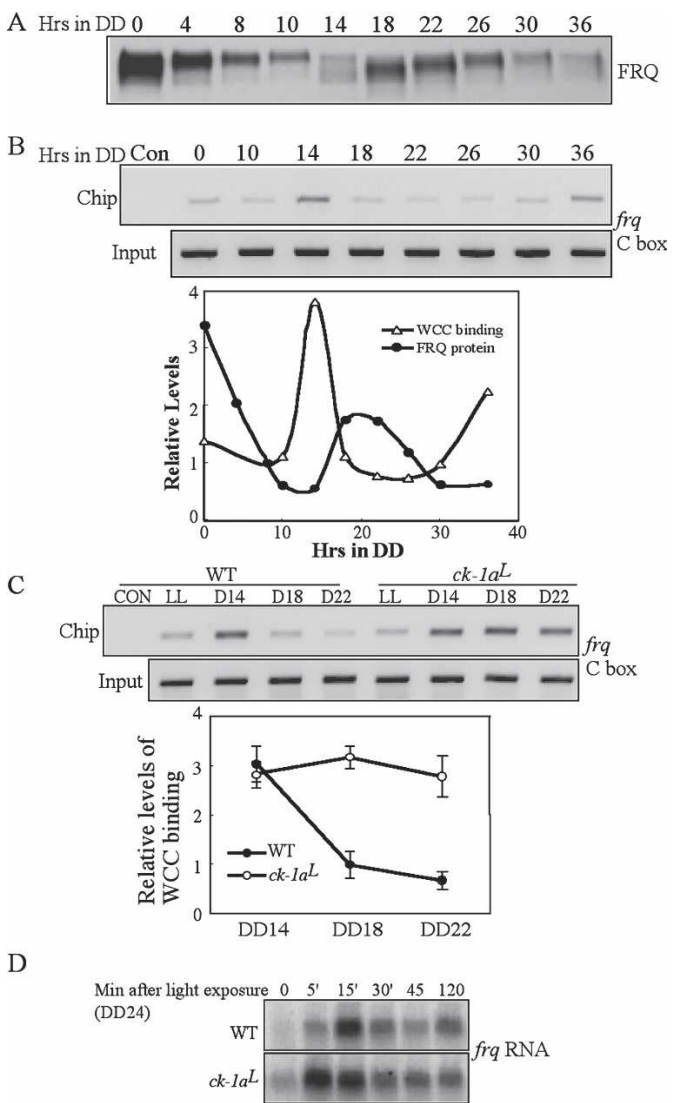

Figure 6. WCC binds to the frq C-box rhythmically in DD in the wild-type strain and the binding cannot be effectively inhibited by FRQ in the $c k-1 a^{L}$ strain. $(A)$ Western blot analysis showing the rhythmic FRQ expression in DD in the wild-type strain. (B) ChIP assay using WC-2 antibody showing that the rhythmic binding of WCC to the frq C-box. The densitometric analysis of the Western and ChIP results is shown below. $(C)$ ChIP assay showing the WCC binding to the frq C-box in the wild-type and $c k-1 a^{L}$ strains at different time points. The densitometric analysis of the ChIP results is shown below. $(D)$ Northern blot analysis showing the light-induced frq transcription in the wild-type and $c k-1 a^{L}$ strains. The cultures were grown in DD for $24 \mathrm{~h}$ before they were transferred into LL.

RNA suggest that FRQ cannot inhibit WCC activity effectively in this mutant. To test this hypothesis, we performed side-by-side comparison of the WCC binding to the frq C-box in the wild-type and mutant strains. As shown in Figure 6C, unlike in the wild type, in which the binding rapidly decreased at DD18, WCC binding to the C-box stayed at a high level for at least $8 \mathrm{~h}$ in the $c k-1 a^{L}$ strain despite the presence of high levels of FRQ (Fig. 5A). These data suggest that the circadian negative feedback loop is severely compromised in the mutant, resulting from the inability for the mutant CK-1a to efficiently phosphorylate WC proteins.

We have previously shown that the light-induced WCC phosphorylation inhibits its role as the transcription factor for light-induced transcription (He and Liu 2005). The hypophosphorylated WC proteins in LL in the $c k-1 a^{L}$ strain suggest that CK-1a also partially contrib- utes to the light-induced WC phosphorylation. Thus, we expected that the kinetics of light-induced transcription would be altered in the mutant. To further demonstrate that the hypophosphorylated WCC is the active form of WCC, we examined the light induction of $f r q$ transcription in the $c k-1 a^{L}$ strain (Fig. 6D). As previously shown for a wild-type strain (Crosthwaite et al. 1995; He and Liu 2005), although a low level of light-induced frq RNA could be seen 5 min after the light exposure, fr $q$ peaked in 15-30 min and decreased afterward as a result of photoadaptation (He and Liu 2005). In contrast, in the $c k-1 a^{L}$ strain, the light-induced $f_{r} q$ RNA reached its peak at a level that was higher than that in the wild-type peak level in only $5 \mathrm{~min}$ and decreased slowly afterward, which indicates that the photoadaptation process is partially impaired in the mutant. These results and the low levels of WC proteins in the $c k-1 a^{L}$ strain suggest that the hypophosphorylated WCC is also the active form that mediates the light-induced transcription. Therefore, CK-1a also plays a role in light response and photoadaptation in Neurospora.

Disruption of cka, the catalytic subunit gene of CKII, results in hypophosphorylation of WC proteins and the failure for FRQ to inhibit WCC activity

The slow decrease of $f r q$ mRNA in the $c k-1 a^{L}$ strain suggests that an additional mechanism is involved in the inhibition of WCC by FRQ (Fig. 5C). The presence of light-dependent WC phosphorylation forms in the $c k$ $1 a^{L}$ strain (Fig. 5B) indicates that an additional kinase(s) is involved in WC phosphorylation. Previously, we identified CKII as a major kinase that phosphorylates FRQ in vivo. The disruption of its catalytic subunit, cka, and one of its regulatory subunits showed that not only FRQ stability is affected in these mutants, the negative feedback loop is also severely impaired (Yang et al. 2002, 2003).

To understand how CKII functions in this process, we examined the phosphorylation profiles of $\mathrm{WC}-1$ and WC-2 in the $c k a^{R I P}$ mutant. As shown in Figure 7A, both WC-1 and WC-2 were hypophosphorylated in DD and LL in the $c k a^{R I P}$ mutant, suggesting that CKII is another kinase that may mediate the phosphorylation of WC-1 and WC-2. However, unlike the $c k-1 a^{L}$ strain, the WC levels were not decreased in the $c k a^{R I P}$ mutant, indicating that CKII is not involved in the regulation of the positive feedback loops. In addition, as previously shown, the FRQ levels were high and hypophosphorylated in the $c k a^{R I P}$ mutant (Yang et al. 2002).

To investigate whether the hypophosphorylated WCs in the $c k a^{R I P}$ mutant is also the result of the inability of FRQ to inhibit WCC activity, we compared WCC C-box binding in the wild-type and mutant strains. As shown in Figure $7 \mathrm{~B}$, whereas the WCC binding in DD in the wild-type strain exhibited a similar behavior as shown earlier, in the mutant, WCC binding to the C-box stayed at a higher level at these time points despite the presence of large amounts of FRQ. Thus, FRQ cannot efficiently inhibit WCC activity in the absence of CKII. Although 
He et al.

A

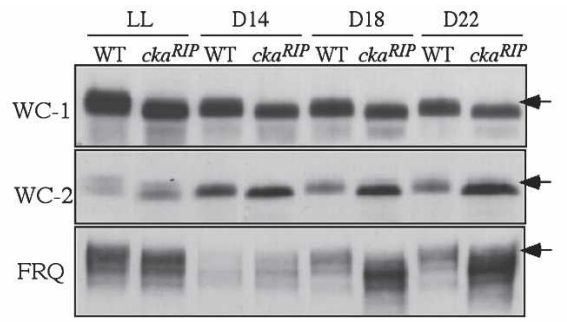

$\mathrm{B}$

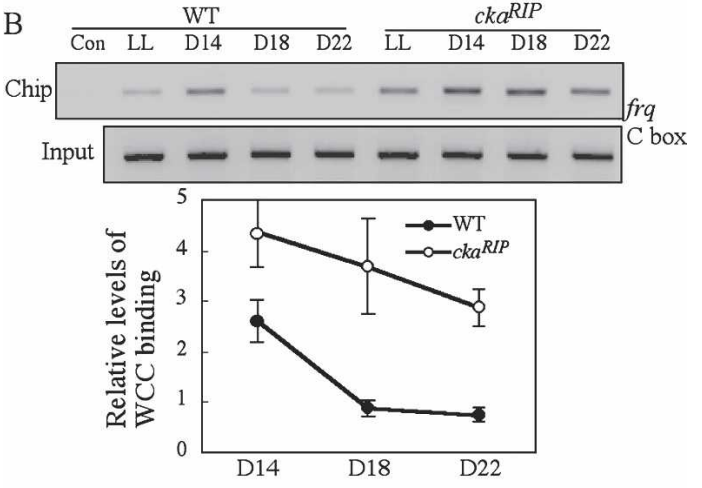

C Min after light exposure $0 \quad 5^{\prime} \quad 15^{\prime} \quad 30^{\prime} \quad 60$ (DD24)

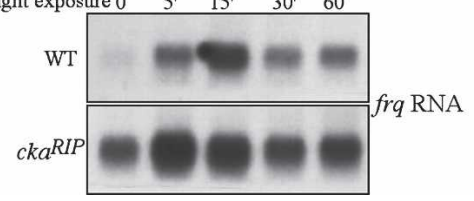

Figure 7. WC-1 and WC-2 are hypophosphorylated and WCC binds to the C-box at constant high levels in the $c k a^{R I P}$ strain. (A) Western blot analyses showing that phosphorylation profiles of WC-1 and WC-2 in the wild-type and $c k a^{R I P}$ strains. The arrows indicate the extensively phosphorylated WC-1, WC-2, and FRQ forms absent in the mutant strain. $(B)$ ChIP assay showing the WCC binding to the frq C-box in the wild-type and $c k a^{R I P}$ strains at different time points. The densitometric analysis of ChIP results is shown below. $(C)$ Northern blot analysis showing the light-induced frq transcription in the wild-type and $c k a^{R I P}$ strains. The cultures were grown in DD for $24 \mathrm{~h}$ before they were transferred into LL.

CKII does not associate with FRQ tightly (Yang et al. 2002, 2003), the transient interactions between FRQ and CKII during the FRQ phosphorylation process will bring CKII to the vicinity of the WC proteins through the FRQ-WCC interaction. Thus, similar to CK-Ia, CKII may also mediate the phosphorylation of WCC in a FRQdependent manner.

We then examined whether the hypophosphorylated WCs in $c k a^{R I P}$ mutant also resulted in changes in the kinetics of light-induced $f r q$ transcription. As shown in Figure 7C, similar to the $c k-1 a^{L}$ strain, the light-induced frq levels peaked at $5 \mathrm{~min}$ in the $c k a^{R I P}$ mutant and elevated to a higher level than that in the wild-type strain. These data suggest that the hypophosphorylated WCC in $c k a^{R I P}$ mutant is also the active form of WCC for clock function and light response and that CKII also plays a role in regulating WC activity after light exposure.

\section{Discussion}

In this study, our results suggest that CK-1a and CKII are not only two of the major kinases phosphorylating FRQ, they are also the key kinases that mediate the FRQ-dependent phosphorylation of WCC to close the circadian negative feedback loop. In addition, CK-1a is also important for the FRQ-dependent accumulation of WC-1 and WC-2.

CK-1a phosphorylates FRQ in vivo, which promotes FRQ degradation and is important for the circadian positive feedback loops

Previously, CKII was the only known major FRQ-phosphorylating kinase in vivo (Yang et al. 2002, 2003). Although CK-1a was shown to associate with FRQ and can phosphorylate FRQ in vitro, its in vivo role in FRQ phosphorylation is not known (Gorl et al. 2001; Cheng et al. 2005; Schafmeier et al. 2006). Here we presented strong evidence indicating that CK-Ia is a kinase that phosphorylates FRQ in vivo. Deletions or mutations of FCD that disrupt the interaction between FRQ and CK-1a result in the hypophosphorylation of FRQ, indicating that significant numbers of FRQ phosphorylation events require the FRQ/CK-1a interaction. More importantly, FRQ is hypophosphorylated in the $c k-1 a^{L}$ strain and the process of FRQ progressive phosphorylation is significantly delayed in the mutant. Similar to CKI's function in Drosophila and mammals (Price et al. 1998; Akashi et al. 2002; Eide et al. 2005a), the phosphorylation of FRQ by CK-1a promotes FRQ degradation. Since FRQ stability is a major determinant for the period length of the clock in Neurospora (Liu et al. 2000; Gorl et al. 2001; Yang et al. 2003), the increased stability of FRQ and the significantly delayed FRQ progressive phosphorylation process resulted in the 32 -h circadian rhythms in the $c k-1 a^{L}$ strain.

Interestingly, three previously identified FRQ phosphorylation sites and the PEST-1 domain are located immediately downstream from the FCD (Liu et al. 2000; Gorl et al. 2001), suggesting that they are likely phosphorylated by CK-1a in vivo. It is also evident that significant amounts of FRQ phosphorylation remain in the FCD mutants and in the $c k-1 a^{L}$ strain, indicating the involvement of other kinases, such as CKII and CAMK-1 (Yang et al. 2001, 2002, 2003). However, it is also possible that some of the FRQ phosphorylation events in the FCD mutant may be mediated by CK-1a independent of the tight FRQ/CK-1a interaction.

FRQ promotes the accumulation of both WC-1 and WC-2, forming the positive feedback loop that is important for robustness and function of the circadian clock in Neurospora (Lee et al. 2000; Cheng et al. 2001b; Schafmeier et al. 2006). Mutation of the in vitro FRQ phosphorylation sites by CK-1a in the PEST-2 domain suggests that these phosphorylation events are important for the accumulation of WC-1, but not WC-2 (Schafmeier et al. 2006). Our results presented here showed that CK-1a is essential for the circadian positive 
feedback loops in vivo. The levels of both WC-1 and WC-2 are low in the FCD mutants and in the $c k-1 a^{L}$ strain (Figs. 2, 3, 5). These results suggest that the phosphorylation of FRQ by CK-1a is not only important for the accumulation of WC-1 but also important for the expression of WC-2. Furthermore, the role of CK-1a in WCC phosphorylation (discussed below) raises the possibility that such post-translational modifications may also contribute to the accumulation of WC proteins. Consistent with this notion, mutations of the identified WC-1 phosphorylation sites near its $\mathrm{Zn}$ finger region result in low WC-1 levels (He et al. 2005b).

In contrast, although CKII is also a major kinase phosphorylating FRQ in vivo and shares similar functions as CK-1a in FRQ degradation and in the circadian negative feedback loop, it is not required for the circadian positive feedback loops (Fig. 7; Yang et al. 2002). This functional difference between CK-1a and CKII suggests that they may phosphorylate functionally distinct sites on FRQ or WCs.

\section{CK-Ia and CKII mediates the FRQ-dependent WCC phosphorylation to close the circadian negative feedback loop}

Our results presented here suggest that CK-1a and CKII mediates the FRQ-dependent WC phosphorylation and inhibits WCC C-box-binding activity. For CK-1a, FRQ mediates CK-1a's phosphorylation of WCC through its association with CK-1a and WCC. First, the disruption of the FRQ/CK-1a interaction by deletion or point mutations of FCD results in the hypophosphorylation of WC-1 and WC-2 (Fig. 2B,C), indicating that the FRQ/CK1a interaction is required for the normal phosphorylation of WCs. Second, CK-1a and WC-1 are present in the same complex in a FRQ-dependent manner (Fig. 2A). Finally, in the $c k-1 a^{L}$ strain, WC-1 and WC-2 are hypophosphorylated despite the presence of partial functional CK1a (Figs. 3D, 5B).

WC-1 and WC-2 are also hypophosphorylated in the cka mutants (Fig. 7A), suggesting that CKII may be another kinase mediating the phosphorylation of WCs. Unlike CK-1a, CKII does not tightly associate with FRQ (Yang et al. 2002, 2003), indicating that their interaction is weak and transient, as most kinase-substrate interactions. Similarly, CKII and WCC were not found to be tightly associated with each other by immunoprecipitation (data not shown). The transient interaction between FRQ and CKII during the FRQ phosphorylation process may bring CKII to the proximity of WCC through the FRQ-WCC interaction. Thus, CKII may also mediate the WC phosphorylation in a FRQ-dependent manner. It is also possible, however, that CKII may mediate some of the WC phosphorylation events independent of FRQ.

Taken together, these results suggest that FRQ acts as a kinase substrate-recruiting subunit to mediate the phosphorylation of WCs by CK-1a and CKII (Fig. 8). What is the role of WC phosphorylation by these two kinases? The ChIP assays showed that in a wild-type strain, WCC binds to the frq C-box rhythmically. The

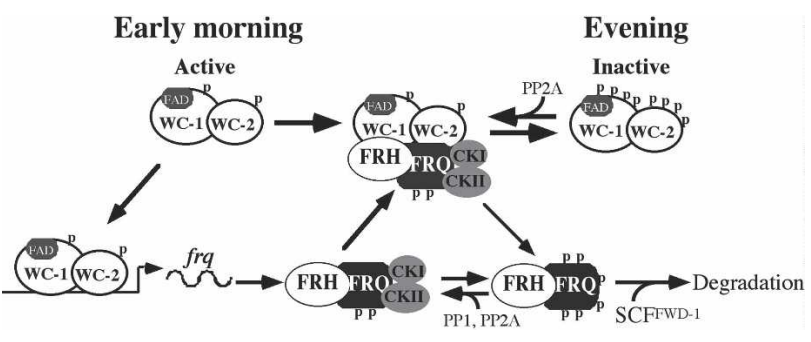

Figure 8. An updated model of the Neurospora circadian negative feedback loop. See the text for details.

peaks of WCC binding occur when FRQ level is at its trough, but the binding is quickly inhibited by the increase of FRQ amounts and stays at a low level for most of the day. In contrast, the inhibition of WCC C-box binding by FRQ is severely impaired in the $c k-1 a^{L}$ and cka mutants (Figs. 6, 7). In the $c k-1 a^{L}$ strain, despite the low levels of WC proteins, WCC binds to the C-box at a high level and could not be inhibited by FRQ even when FRQ level is high. In the $c k a^{R I P}$ mutant, WCC binding at the C-box persists at a higher than wild-type level in DD despite its significantly higher than wild-type FRQ level, resulting in high frq RNA levels. The enhanced WCC activity in the $c k-1 a^{L}$ and $c k a$ mutants is further indicated by the rapid and elevated light-induced frq transcription in these strains. Thus, these results indicate that the phosphorylation of the WC proteins by these two kinases inhibits WCC's activity and is essential for the circadian negative feedback loop.

The involvement of both CK-1a and CKII in WCC phosphorylation suggests that these two kinases may act collaboratively. CKI and CKII have distinct substrate consensus sequences: (D/E/S $\left./ \mathrm{S}^{\mathrm{p}} / \mathrm{T}\right) \mathrm{XX}(\underline{\mathrm{S}} / \mathrm{T})$ for $\mathrm{CKI}$ and $(\underline{\mathrm{S}} /$ $\underline{\mathrm{T}}) \mathrm{XX}\left(\mathrm{D} / \mathrm{E} / \mathrm{S}^{\mathrm{p}} / \mathrm{T}^{\mathrm{p}}\right)$ for CKII (p stands for a phosphorylated residue). Thus, it is possible that they each phosphorylate distinct sites on WC proteins. On the other hand, since they both prefer sites surrounded by acidic or phosphorylated residues, it is also likely that one kinase may act as a priming kinase for the other (or other unknown kinases) to achieve proper phosphorylation of WC proteins, similar to the case suggested by the mutation in the human familial advanced sleep phase syndrome (FASPS) (Toh et al. 2001). The WC sites phosphorylated by CK-1a and CKII are currently unknown. Previously, we identified five major in vivo WC-1 phosphorylation sites located immediately downstream from its DNAbinding domain (He et al. 2005b). Consistent with the results presented here, mutation of these sites suggests that phosphorylation negatively regulates the function of WCC. However, these sites do not resemble the typical CKI or CKII consensus sites and two of the phosphorylated serines are followed by a proline residue. Although CKI and CKII are not commonly known as proline-directed kinases, proline-directed phosphorylation by CKI in vivo has been reported (Alappat et al. 2005). Thus, it is possible that CK-1a may be a kinase that can phosphorylate these five WC-1 sites in vivo.

Figure 8 presents an updated model of the Neurospora 
circadian feedback loop based on this and previous studies with an emphasis on WC phosphorylation. In the subjective early morning in DD, FRQ level is low and WCs are hypophosphorylated. The hypophosphorylated WCC is active and it binds the C-box to activate frq transcription (Cheng et al. 2001b; Froehlich et al. 2002, 2003). After FRQ protein is made, the homodimeric FRQ forms a complex FRH and associates with CK-1a (tightly) (Cheng et al. 2001a, 2005; Gorl et al. 2001) and CKII (weakly). Afterward, FFC interacts with WCC and brings CK-1a and CKII to the proximity of WCC to phosphorylate the WC proteins (Cheng et al. 2001a; Denault et al. 2001). When FRQ level is high, this results in the extensive phosphorylation and inactivation of WCC (He and Liu 2005; He et al. 2005b; Schafmeier et al. 2005, 2006). This process removes WCC from the frq promoter and results in the decrease of $f r q$ transcription. At the same time, FRQ is progressively phosphorylated by CK-1a and CKII and becomes extensively phosphorylated. The extensively phosphorylated FRQ is then recognized by the $\mathrm{SCF}^{\mathrm{FWD}-1} \mathrm{E} 3$ ubiquitin ligase, leading to the ubiquitination and degradation of FRQ through the proteasome pathway (He et al. 2003, 2005a). After FRQ decreases to a certain level, the inactive hyperphosphorylated WCC is dephosphosphorylated by PP2A and probably other PPases (Schafmeier et al. 2005), which results in the reactivation of WCC and frq transcription in a new circadian cycle. In this model, FRQ amount determines the amount of kinases that can be recruited to phosphorylate WCC, and thus, the extent of WCC phosphorylation and its activity. Therefore, the oscillation of FRQ is essential for the function of this circadian negative feedback loop, and the stability of FRQ plays a major role in period length determination (Liu et al. 2000; Gorl et al. 2001; Yang et al. 2003; Ruoff et al. 2005).

\section{Conservation of post-translational regulation in the eukaryotic circadian systems}

The conservation of the post-translational mechanisms is remarkable among different eukaryotic circadian systems from fungi to humans (Heintzen and Liu 2006). Like FRQ and WCs, the animal and plant core clock components are also regulated by phosphorylation (Young and Kay 2001). FRQ and the animal PER proteins are all phosphorylated by CKI and CKII, dephosphorylated by the same phosphatases, and degraded by the ubiquitin/proteasome system using a conserved E3 ubiquitin ligase (Kloss et al. 1998; Price et al. 1998; Lowrey et al. 2000; Gorl et al. 2001; Lee et al. 2001; Grima et al. 2002; Ko et al. 2002; Lin et al. 2002; Yang et al. 2002, 2003, 2004; Akten et al. 2003; He et al. 2003; Nawathean and Rosbash 2004; Sathyanarayanan et al. 2004; Eide et al. 2005b; Xu et al. 2005). Like in Neurospora, CKI was also found to be tightly associated with PER proteins in Drosophila and mammals (Kloss et al. 1998; Lee et al. 2001, 2004). In mouse, the PER/CKI interaction was suggested to be a critical process in regulating $\mathrm{MPER}$ phosphorylation and the function of the clock (Lee et al. 2004). Similar to the role of CKI in animal clock systems
(Price et al. 1998; Akashi et al. 2002; Eide et al. 2005a), we showed here that the $c k-1 a^{L}$ strain, which carries the same mutation as the Drosophila long period $d b t^{L} \mathrm{mu}-$ tants, exhibiting long period rhythms and impaired FRQ degradation. In humans, two types of Familial Advanced Sleep Phase Syndrome (FASPS) were found to be due to mutations of the human CKIS or its phoshorylation sites on hPER2 (Toh et al. 2001; Xu et al. 2005). For CKII, like we showed in Neurospora, it was also found to be required for the PER repressor function in Drosophila (Nawathean and Rosbash 2004), although how CKII functions to promote the repressor activity of PER is not clear. In Arabidopsis, where CKII was first implicated in clock function, it has been shown to phosphorylate CCA1 and regulate its DNA-binding activity (Sugano et al. 1998).

Like the WC proteins, the PAS-domain-containing transcriptional factors that act as the positive limbs of the animal circadian negative feedback loops are also regulated by phosphorylation, and the mammalian BMAL1 and CLK and the Drosophila CLK also exhibit robust rhythms in their phosphorylation profiles (Lee et al. 2001; Kim and Edery 2006; Yu et al. 2006). More remarkably, resembling the situation in Neurospora, the phosphorylation of CLK was recently found to be dependent on PER and may be mediated by DBT (Lee et al. 2001; Kim and Edery 2006; Yu et al. 2006). In addition, the CLK-CYC binding to the per E-box correlates with the accumulation of hypophosphorylation of CLK, and the hyperphosphorylation of CLK correlates with the peak of per mRNA repression (Kim and Edery 2006; Yu et al. 2006). Furthermore, PP2A was also shown to be involved in the regulation of CLK phosphorylation (Kim and Edery 2006). In cultured S2 cells, DBT-dependent CLK phosphorylation promotes CLK degradation. Although the role of CLK phosphorylation by DBT in the regulation of CLK-CYC DNA-binding activity is still lacking and the mechanism for how CKII works is not known in Drosophila, our results presented in this study and the conservation of the circadian systems suggest that a similar mechanism for the closing of the negative feedback loop exists in Neurospora, Drosophila, and probably mammals.

\section{Materials and methods}

Strains and culture conditions

87-3 (bd a) was used as the wild-type strain in this study. The $k u-70^{R I P}$ and $c k-1 a^{L}$ strains were created in this study. The $c k-$ $a^{R I P}$ strain, in which the cka gene was disrupted by multiple premature stop codons, was generated previously (Yang et al. 2002). The 303-3 (frq ${ }^{10}$, his-3) strain was the host strain for the his-3 targeting frq constructs. Various sFRQ strains that express sFRQ with internal in-frame deletions were created previously (Liu et al. 2000; Cheng et al. 2005). Liquid cultures were grown

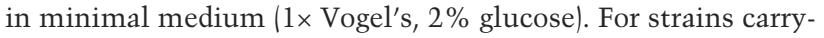
ing FRQ and Myc-CK-1a constructs, liquid cultures were grown in $0.01 \mathrm{M}$ QA (pH 5.8), 1× Vogel's, $0.1 \%$ glucose, and $0.17 \%$ arginine. Race tube medium contains $1 \times V_{\text {Vogel's, }} 0.1 \%$ glucose, $0.17 \%$ arginine, $50 \mathrm{ng} / \mathrm{mL}$ biotin, and $1.5 \%$ agar. The densito- 
metric analyses of the race tubes were performed using NIH Image.

\section{Creation of frq mutant strains to examine FRQ/} CK-1a interaction

pKAJ120 (containing the entire fr $q$ gene including its promoter and a his-3 targeting sequence) is the parental plasmid for all frq constructs described in this study. All of the deletion and point mutations of the FRQ ORF were constructed using either the Transformer Site-Directed Mutagenesis Kit (Clontech Laboratories) or QuikChange Site-Directed Mutagenesis Kit (Stratagene), and pUC19Mfrq was used as the in vitro mutagenic template. Afterward, the mutations were subcloned into pKAJ120, and the resulting constructs were transformed into the $f r q^{10}$, his-3 at the his-3 locus by electroporation (Margolin et al. 1999). To study FRQ/CK-1a interaction, the Myc-CK-1a construct was introduced into the transformants of various FRQ mutants by cotransformation with pBT6 /containing the benomyl resistance gene, obtained from Fungal Genetic Stock Center). The Myc-CK-1a construct was made by inserting a PCR fragment, which contains the CK-1a ORF and its 3'UTR, into pqa.5Myc vector (He et al. 2003).

Creation of the $\mathrm{ku} 70^{\text {RIP }}$ strain

The Neurospora ku70 gene (Ninomiya et al. 2004) was disrupted by repeat-induced point mutation (RIP) (Cambareri et al. 1989). The PCR fragment containing the entire ku70 ORF and its 3'UTR was cloned into $\mathrm{pDE} 3 \mathrm{BH}$ and introduced into the his-3 locus of a wild-type strain (bd his-3 A) by electroporation. After the identification of positive transformants, they were crossed with another wild-type strain $(b d a)$. Southern blot analysis was used to identify progenies with a mutated ku70 gene. Afterward, DNA sequencing of the endogenous ku70 was performed to identify the strains in which the endogenous ku70 ORF was mutated with multiple premature stop codons. The resulting $b d$ $k u 70^{R I P}$ strain was used as the host strain for introducing the ck-1a knock-in cassette.

\section{Creation of the ck-1 $a^{L}$ strains}

To create the knock-in cassette with the $d b t^{L}$ mutation, the wild-type $c k-1 a$ gene was cloned into $\mathrm{pDE} 3 \mathrm{BH}$ and the resulting plasmid, pCKI-2, was used as the template for in vitro mutagenesis to mutate the CK-1a Met83 to Ile. Afterward, a ligationPCR approach was used to generate the knock-in cassette using the mutated $c k-1 a$ gene and pCN44 (containing the $h p h$ resistance gene) as PCR templates (Zhao et al. 2004). In the resulting knock-in PCR fragment, the $h p h$ gene is inserted downstream from the $c k-1 a$ 3'UTR and flanked by the entire $c k-1 a$ gene (with the mutation) and $0.5 \mathrm{~kb}$ genomic DNA downstream from $c k-1 a$ (see Fig. 3A). The cassette was then transformed into the bd ku $70^{R I P}$ strain to select for $h p h$-resistant transformants. The genomic DNA from the transformants was prepared, and PCR was used to identify transformants with $h p h$ integrated into the endogenous $c k-1 a$ locus. PCR products containing the endogenous $c k-1 a$ were sequenced to identify the heterokaryon strains with the knock-in mutation. Afterward, the homokaryotic $c k-1 a^{L}$ strains were obtained by microconidia purification (Ebbole and Sachs 1990) and confirmed by Southern blot analysis and DNA sequencing.

\section{Protein and RNA analyses}

Protein extraction, quantification, Western blot analysis, and immunoprecipitation assays were performed as previously de- scribed (Garceau et al. 1997; Cheng et al. 2001a). Equal amounts of total protein $(40 \mu \mathrm{g})$ were loaded in each protein lane of SDSPAGE, and after electrophoresis, proteins were transferred onto PVDF membrane, and Western blot analyses were performed. To analyze the phosphorylation profiles of WC-1 and WC-2, $10 \%$ SDS-PAGE gels containing a ratio of 149:1 acrylamide/ bisacrylamide were used. Otherwise, 7.5\% SDS-PAGE gels containing a ratio of 37.5:1 acrylamide/bisacrylamide were used.

RNA extraction and Northern blot analyses were performed as described previously (Aronson et al. 1994). Equal amounts of total RNA $(20 \mu \mathrm{g})$ were loaded onto agarose gels for electrophoresis, and the gels were blotted and probed with an RNA probe specific for frq.

\section{ChIP}

The ChIP assays protocol and the primers used are as previously described (He and Liu 2005). PCR reactions were as follows: 4 min at $94^{\circ} \mathrm{C}, 26-29$ cycles of $94^{\circ} \mathrm{C}(15 \mathrm{sec}), 60^{\circ} \mathrm{C}(30 \mathrm{sec})$, and $72^{\circ} \mathrm{C}(1 \mathrm{~min})$. Different PCR cycle numbers were tested to ensure that DNA amplification was within the exponential amplification range. PCR products were resolved by electrophoresis on $2 \%$ agarose gels. Each experiment was independently performed three times, and immunoprecipitation without the WC-2 antibody was used as the negative controls.

\section{Acknowledgments}

We thank Lixin Wang for excellent technical assistance and Dr. Lily Li for critical reading and editing of the manuscript. This study was supported by grants from National Institutes of Health (GM062591 and GM068496) and Welch Foundation to YL. Y.L. is the Louise W. Kahn endowed scholar in Biomedical Research at University of Texas Southwestern Medical Center.

\section{References}

Akashi, M., Tsuchiya, Y., Yoshino, T., and Nishida, E. 2002. Control of intracellular dynamics of mammalian period proteins by casein kinase I $\varepsilon(\mathrm{CKI} \varepsilon)$ and CKI $\delta$ in cultured cells. Mol. Cell. Biol. 22: 1693-1703.

Akten, B., Jauch, E., Genova, G.K., Kim, E.Y., Edery, I., Raabe, T., and Jackson, F.R. 2003. A role for CK2 in the Drosophila circadian oscillator. Nat. Neurosci. 6: 251-257.

Alappat, E.C., Feig, C., Boyerinas, B., Volkland, J., Samuels, M., Murmann, A.E., Thorburn, A., Kidd, V.J., Slaughter, C.A., Osborn, S.L., et al. 2005. Phosphorylation of FADD at serine 194 by CKI $\alpha$ regulates its nonapoptotic activities. Mol. Cell 19: 321-332.

Aronson, B., Johnson, K., Loros, J.J., and Dunlap, J.C. 1994. Negative feedback defining a circadian clock: Autoregulation in the clock gene frequency. Science 263: 1578-1584.

Cambareri, E.B., Jensen, B.C., Schabtach, E., and Selker, E.U. 1989. Repeat-induced G-C to A-T mutations in Neurospora. Science 244: 1571-1575.

Cheng, P., Yang, Y., Heintzen, C., and Liu, Y. 2001a. Coiled-coil domain mediated FRQ-FRQ interaction is essential for its circadian clock function in Neurospora. EMBO J. 20: 101108.

Cheng, P., Yang, Y., and Liu, Y. 2001b. Interlocked feedback loops contribute to the robustness of the Neurospora circadian clock. Proc. Natl. Acad. Sci. 98: 7408-7413.

Cheng, P., Yang, Y., Gardner, K.H., and Liu, Y. 2002. PAS domain-mediated WC-1/WC-2 interaction is essential for maintaining the steady state level of WC-1 and the function 
of both proteins in circadian clock and light responses of Neurospora. Mol. Cell. Biol. 22: 517-524.

Cheng, P., Yang, Y., Wang, L., He, Q., and Liu, Y. 2003. WHITE COLLAR-1, a multifunctional Neurospora protein involved in the circadian feedback loops, light sensing, and transcription repression of wc-2. J. Biol. Chem. 278: 3801-3808.

Cheng, P., He, Q., He, Q., Wang, L., and Liu, Y. 2005. Regulation of the Neurospora circadian clock by an RNA helicase. Genes \& Dev. 19: 234-241.

Crosthwaite, S.K., Loros, J.J., and Dunlap, J.C. 1995. Light-Induced resetting of a circadian clock is mediated by a rapid increase in frequency transcript. Cell 81: 1003-1012.

Crosthwaite, S.K., Dunlap, J.C., and Loros, J.J. 1997. Neurospora $w c-1$ and $w c-2$ : Transcription, photoresponses, and the origins of circadian rhythmicity. Science 276: 763-769.

Denault, D.L., Loros, J.J., and Dunlap, J.C. 2001. WC-2 mediates WC-1-FRQ interaction within the PAS protein-linked circadian feedback loop of Neurospora. EMBO J. 20: 109-117.

Dunlap, J.C. 1999. Molecular bases for circadian clocks. Cell 96: 271-290.

Ebbole, D. and Sachs, M.S. 1990. A rapid and simple method for isolation of Neurospora crassa homokaryons using microconidia. Fungal Genet. Newsl. 37: 17-18.

Eide, E.J., Woolf, M.F., Kang, H., Woolf, P., Hurst, W., Camacho, F., Vielhaber, E.L., Giovanni, A., and Virshup, D.M. 2005a. Control of mammalian circadian rhythm by CKI $\varepsilon$-regulated proteasome-mediated PER2 degradation. Mol. Cell. Biol. 25: 2795-2807.

- 2005b. Control of mammalian circadian rhythm by CKI $\varepsilon$-regulated proteasome-mediated PER2 degradation. Mol. Cell. Biol. 25: 2795-2807.

Froehlich, A.C., Liu, Y., Loros, J.J., and Dunlap, J.C. 2002. White Collar-1, a circadian blue light photoreceptor, binding to the frequency promoter. Science 297: 815-819.

Froehlich, A.C., Loros, J.J., and Dunlap, J.C. 2003. Rhythmic binding of a WHITE COLLAR-containing complex to the frequency promoter is inhibited by FREQUENCY. Proc. Nat1. Acad. Sci. 100: 5914-5919.

Garceau, N., Liu, Y., Loros, J.J., and Dunlap, J.C. 1997. Alternative initiation of translation and time-specific phosphorylation yield multiple forms of the essential clock protein FREQUENCY. Cell 89: 469-476.

Glossop, N.R., Lyons, L.C., and Hardin, P.E. 1999. Interlocked feedback loops within the Drosophila circadian oscillator. Science 286: 766-768.

Gorl, M., Merrow, M., Huttner, B., Johnson, J., Roenneberg, T., and Brunner, M. 2001. A PEST-like element in FREQUENCY determines the length of the circadian period in Neurospora crassa. EMBO I. 20: 7074-7084.

Grima, B., Lamouroux, A., Chelot, E., Papin, C., LimbourgBouchon, B., and Rouyer, F. 2002. The F-box protein slimb controls the levels of clock proteins period and timeless. Nature 420: 178-182.

He, Q. and Liu, Y. 2005. Molecular mechanism of light responses in Neurospora: From light-induced transcription to photoadaptation. Genes \& Dev. 19: 2888-2899.

He, Q., Cheng, P., Yang, Y., Wang, L., Gardner, K.H., and Liu, Y. 2002. White collar-1, a DNA binding transcription factor and a light sensor. Science 297: 840-843.

He, Q., Cheng, P., Yang, Y., He, Q., Yu, H., and Liu, Y. 2003. FWD1-mediated degradation of FREQUENCY in Neurospora establishes a conserved mechanism for circadian clock regulation. EMBO J. 22: 4421-4430.

He, Q., Cheng, P., He, Q., and Liu, Y. 2005a. The COP9 signalosome regulates the Neurospora circadian clock by controlling the stability of the SCFFWD-1 complex. Genes \& Dev.
19: $1518-1531$.

He, Q., Shu, H., Cheng, P., Chen, S., Wang, L., and Liu, Y. 2005b. Light-independent phosphorylation of WHITE COLLAR-1 regulates its function in the Neurospora circadian negative feedback loop. J. Biol. Chem. 280: 17526-17532.

Heintzen, C. and Liu, Y. 2006. The Neurospora circadian clock. Adv. Genet. (in press).

Kim, E.Y. and Edery, I. 2006. Balance between DBT/CKI $\{$ var $\varepsilon$ kinase and protein phosphatase activities regulate phosphorylation and stability of Drosophila CLOCK protein. Proc. Nat1. Acad. Sci. 103: 6178-6183.

Kloss, B., Price, J.L., Saez, L., Blau, J., Rothenfluh, A., and Young, M.W. 1998. The Drosophila clock gene double-time encodes a protein closely related to human casein kinase Ie. Cell 94: 97-107.

Ko, H.W., Jiang, J., and Edery, I. 2002. Role for Slimb in the degradation of Drosophila Period protein phosphorylated by Doubletime. Nature 420: 673-678.

Lee, K., Loros, J.J., and Dunlap, J.C. 2000. Interconnected feedback loops in the Neurospora circadian system. Science 289: 107-110.

Lee, C., Etchegaray, J.P., Cagampang, F.R., Loudon, A.S., and Reppert, S.M. 2001. Posttranslational mechanisms regulate the mammalian circadian clock. Cell 107: 855-867.

Lee, C., Weaver, D.R., and Reppert, S.M. 2004. Direct association between mouse PERIOD and CKI $\varepsilon$ is critical for a functioning circadian clock. Mol. Cell. Biol. 24: 584-594.

Lin, J.M., Kilman, V.L., Keegan, K., Paddock, B., Emery-Le, M., Rosbash, M., and Allada, R. 2002. A role for casein kinase $2 \alpha$ in the Drosophila circadian clock. Nature 420: 816-820.

Liu, Y. 2005. Analysis of posttranslational regulations in the Neurospora circadian clock. Methods Enzymol. 393: 379393.

Liu, Y., Loros, J., and Dunlap, J.C. 2000. Phosphorylation of the Neurospora clock protein FREQUENCY determines its degradation rate and strongly influences the period length of the circadian clock. Proc. Natl. Acad. Sci. 97: 234-239.

Loros, J.J. and Dunlap, J.C. 2001. Genetic and molecular analysis of circadian rhythms in Neurospora. Annu. Rev. Physiol. 63: 757-794.

Lowrey, P.L., Shimomura, K., Antoch, M.P., Yamazaki, S., Zemenides, P.D., Ralph, M.R., Menaker, M., and Takahashi, J.S. 2000. Positional syntenic cloning and functional characterization of the mammalian circadian mutation tau. Science 288: 483-492.

Margolin, B.S., Freitag, M., and Selker, E.U. 1999. Improved plasmids for gene targeting at the his-3 locus of Neurospora crassa by electroporation. Fungal Genet. Newsl. 44: 24-36.

Merrow, M., Garceau, N., and Dunlap, J.C. 1997. Dissection of a circadian oscillation into discrete domains. Proc. Nat1. Acad. Sci. 94: 3877-3882.

Merrow, M., Franchi, L., Dragovic, Z., Gorl, M., Johnson, J., Brunner, M., Macino, G., and Roenneberg, T. 2001. Circadian regulation of the light input pathway in Neurospora crassa. EMBO I. 20: 307-315.

Nawathean, P. and Rosbash, M. 2004. The doubletime and CKII kinases collaborate to potentiate Drosophila PER transcriptional repressor activity. Mol. Cell 13: 213-223.

Ninomiya, Y., Suzuki, K., Ishii, C., and Inoue, H. 2004. Highly efficient gene replacements in Neurospora strains deficient for nonhomologous end-joining. Proc. Natl. Acad. Sci. 101: $12248-12253$.

Okamura, H., Garcia-Rodriguez, C., Martinson, H., Qin, J., Virshup, D.M., and Rao, A. 2004. A conserved docking motif for CK1 binding controls the nuclear localization of NFAT1. Mol. Cell. Biol. 24: 4184-4195. 
Preuss, F., Fan, J.Y., Kalive, M., Bao, S., Schuenemann, E., Bjes, E.S., and Price, J.L. 2004. Drosophila doubletime mutations which either shorten or lengthen the period of circadian rhythms decrease the protein kinase activity of casein kinase I. Mol. Cell. Biol. 24: 886-898.

Price, J.L., Blau, J., Rothenfluh, A., Adodeely, M., Kloss, B., and Young, M.W. 1998. double-time is a new Drosophila clock gene that regulates PERIOD protein accumulation. Cell 94: 83-95.

Reppert, S.M. and Weaver, D.R. 2001. Molecular analysis of mammalian CIRCADIAN rhythms. Annu. Rev. Physiol. 63: 647-676.

Ripperger, J.A. and Schibler, U. 2006. Rhythmic CLOCKBMAL1 binding to multiple E-box motifs drives circadian Dbp transcription and chromatin transitions. Nat. Genet. 38: 369-374.

Ruoff, P., Loros, J.J., and Dunlap, J.C. 2005. The relationship between FRQ-protein stability and temperature compensation in the Neurospora circadian clock. Proc. Natl. Acad. Sci. 102: 17681-17686.

Sathyanarayanan, S., Zheng, X., Xiao, R., and Sehgal, A. 2004. Posttranslational regulation of Drosophila PERIOD protein by protein phosphatase 2A. Cell 116: 603-615.

Schafmeier, T., Haase, A., Kaldi, K., Scholz, J., Fuchs, M., and Brunner, M. 2005. Transcriptional feedback of neurospora circadian clock gene by phosphorylation-dependent inactivation of its transcription factor. Cell 122: 235-246.

Schafmeier, T., Kaldi, K., Diernfellner, A., Mohr, C., and Brunner, M. 2006. Phosphorylation-dependent maturation of Neurospora circadian clock protein from a nuclear repressor toward a cytoplasmic activator. Genes \& Dev. 20: 297-306.

Schwerdtfeger, C. and Linden, H. 2000. Localization and lightdependent phosphorylation of white collar 1 and 2, the two central components of blue light signaling in Neurospora crassa. Eur. J. Biochem. 267: 414-422.

Sehgal, A. 2004. Molecular biology of circadian rhythms. John Wiley \& Sons, Hoboken, NJ.

Shearman, L.P., Sriram, S., Weaver, D.R., Maywood, E.S., Chaves, I., Zheng, B., Kume, K., Lee, C.C., van der Horst, G.T., Hastings, M.H., et al. 2000. Interacting molecular loops in the mammalian circadian clock. Science 288: 10131019.

Sugano, S., Andonis, C., Green, R., Wang, Z.Y., and Tobin, E.M. 1998. Protein kinase CK2 interacts with and phosphorylates the Arabidopsis circadian clock-associated 1 protein. Proc. Natl. Acad. Sci. 95: 11020-11025.

Talora, C., Franchi, L., Linden, H., Ballario, P., and Macino, G. 1999. Role of a white collar-1-white collar-2 complex in blue-light signal transduction. EMBO J. 18: 4961-4968.

Toh, K.L., Jones, C.R., He, Y., Eide, E.J., Hinz, W.A., Virshup, D.M., Ptacek, L.J., and Fu, Y.H. 2001. An hPer2 phosphorylation site mutation in familial advanced sleep phase syndrome. Science 291: 1040-1043.

$\mathrm{Xu}$, Y., Padiath, Q.S., Shapiro, R.E., Jones, C.R., Wu, S.C., Saigoh, N., Saigoh, K., Ptacek, L.J., and Fu, Y.H. 2005. Functional consequences of a CKI $\delta$ mutation causing familial advanced sleep phase syndrome. Nature 434: 640-644.

Yang, Y., Cheng, P., Zhi, G., and Liu, Y. 2001. Identification of a calcium/calmodulin-dependent protein kinase that phosphorylates the Neurospora circadian clock protein FREQUENCY. J. Biol. Chem. 276: 41064-41072.

Yang, Y., Cheng, P., and Liu, Y. 2002. Regulation of the Neurospora circadian clock by casein kinase II. Genes \& Dev. 16: 994-1006.

Yang, Y., Cheng, P., He, Q., Wang, L., and Liu, Y. 2003. Phosphorylation of FREQUENCY protein by casein kinase II is necessary for the function of the Neurospora circadian clock. Mol. Cell. Biol. 23: 6221-6228.

Yang, Y., He, Q., Cheng, P., Wrage, P., Yarden, O., and Liu, Y. 2004. Distinct roles for PP1 and PP2A in the Neurospora circadian clock. Genes \& Dev. 18: 255-260.

Young, M.W. and Kay, S.A. 2001. Time zones: A comparative genetics of circadian clocks. Nat. Rev. Genet. 2: 702-715.

$\mathrm{Yu}$, W., Zheng, H., Houl, J.H., Dauwalder, B., and Hardin, P.E. 2006. PER-dependent rhythms in CLK phosphorylation and E-box binding regulate circadian transcription. Genes \& Dev. 20: 723-733.

Zhao, X., Xue, C., Kim, Y., and Xu, J.R. 2004. A ligation-PCR approach for generating gene replacement constructs in Magnaporthe grisea. Fungal Genet. Newsl. 51: 17-18. 


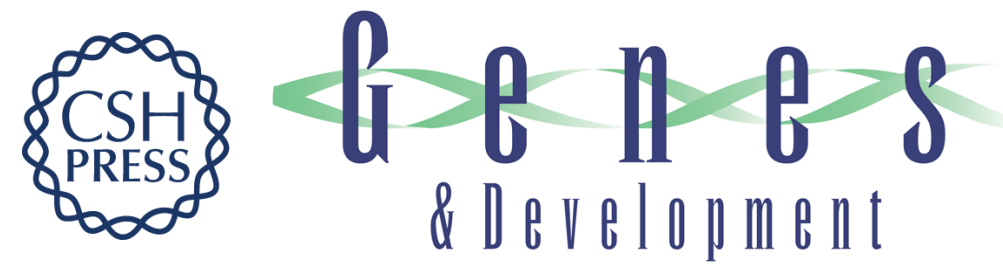

\section{CKI and CKII mediate the FREQUENCY-dependent phosphorylation of the WHITE COLLAR complex to close the Neurospora circadian negative feedback loop}

Qun He, Joonseok Cha, Qiyang He, et al.

Genes Dev. 2006, 20:

Access the most recent version at doi:10.1101/gad.1463506

Supplemental http://genesdev.cshlp.org/content/suppl/2006/09/18/20.18.2552.DC1

Material

References This article cites 66 articles, 42 of which can be accessed free at:

http://genesdev.cshlp.org/content/20/18/2552.full.html\#ref-list-1

License

Email Alerting Receive free email alerts when new articles cite this article - sign up in the box at the top

Service right corner of the article or click here.

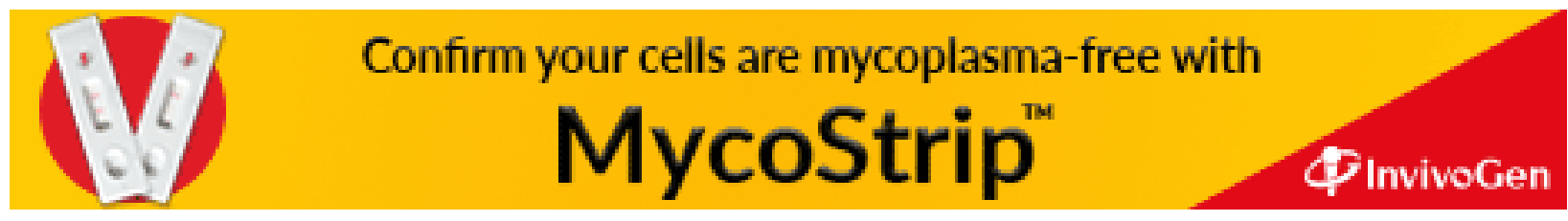

